\title{
Current contribution of diffusion tensor imaging in the evaluation of diffuse axonal injury
}

\author{
Contribuição atual da imagem por tensor de difusão na avaliação da lesão axonal difusa \\ Daphine Centola Grassi', David Macedo da Conceição', Claudia da Costa Leite', Celi Santos Andrade1
}

\begin{abstract}
Traumatic brain injury (TBI) is the number one cause of death and morbidity among young adults. Moreover, survivors are frequently left with functional disabilities during the most productive years of their lives. One main aspect of TBI pathology is diffuse axonal injury, which is increasingly recognized due to its presence in $40 \%$ to $50 \%$ of all cases that require hospital admission. Diffuse axonal injury is defined as widespread axonal damage and is characterized by complete axotomy and secondary reactions due to overall axonopathy. These changes can be seen in neuroimaging studies as hemorrhagic focal areas and diffuse edema. However, the diffuse axonal injury findings are frequently under-recognized in conventional neuroimaging studies. In such scenarios, diffuse tensor imaging (DTI) plays an important role because it provides further information on white matter integrity that is not obtained with standard magnetic resonance imaging sequences. Extensive reviews concerning the physics of DTI and its use in the context of TBI patients have been published, but these issues are still hazy for many allied-health professionals. Herein, we aim to review the current contribution of diverse state-of-the-art DTI analytical methods to the understanding of diffuse axonal injury pathophysiology and prognosis, to serve as a quick reference for those interested in planning new studies and who are involved in the care of TBI victims. For this purpose, a comprehensive search in Pubmed was performed using the following keywords: "traumatic brain injury", "diffuse axonal injury", and "diffusion tensor imaging".
\end{abstract}

Keywords: magnetic resonance imaging; diffusion tensor imaging; diffuse axonal injury; brain injuries, traumatic.

\section{RESUMO}

O traumatismo cranioencefálico (TCE) é a principal causa de morbimortalidade entre adultos jovens. Aqueles que sobrevivem são frequentemente deixados com sequelas funcionais nos anos mais produtivos de suas vidas. 0 principal aspecto fisiopatológico do TCE é a lesão axonial difusa (LAD), cada vez mais destacada pois está presente em 40 a 50\% dos casos que necessitam de internação hospitalar. LAD é definida como a injúria axonial extensa caracterizada pela axoniotomia completa assim como pelas reações secundárias a axoniopatia, que são demonstradas por métodos de neuroimagem como áreas de edema e micro-hemorragia. Entretanto, os achados da LAD são frequentemente subestimados em estudos de neuroimagem convencional. É neste contexto que imagens por tensor de difusão (DTI) ganharam ênfase, já que permitem obter informações sobre a integridade da substância branca que não eram obtidas por sequências convencionais de ressonância magnética (RM). Existem artigos extensos sobre os fundamentos físicos e as aplicações de DTI em pacientes vítimas de TCE, no entanto, estes assuntos permanecem ainda nebulosos a alguns profissionais da área de saúde. Deste modo, propomos uma revisão didática sobre a contribuição do estado da arte de diferentes métodos analíticos de DTI no entendimento do processo da fisiopatologia e prognóstico da LAD, servindo assim como uma ferramenta acessível para aqueles interessados em planejamento de novos estudos e aqueles envolvidos no tratamento de vítimas de TCE. Uma pesquisa abrangente foi realizada no Pubmed com as seguintes palavras-chave: "traumatismo cranioencefálico", "lesão axonial difusa”, "imagem por tensor de difusão".

Palavras-chave: imagem por ressonância magnética; imagem de tensor de difusão; lesão axonal difusa; lesões encefálicas traumáticas

\section{TRAUMATIC BRAIN INJURY}

Currently, traumatic brain injury (TBI) is a worldwide public health problem due to its high prevalence, morbidity and number of deaths. The most affected individuals are young males, who are more likely to engage in risk-taking behaviors. These traumatic events may result in several disabilities, loss of productivity and impaired quality of life. Therefore, understanding the mechanisms of trauma, grading the injury, and providing adequate medical care to the victims are essential to minimize the large social and economic consequences of $\mathrm{TBIs}^{1,2}$.

¿Universidade de São Paulo, Faculdade de Medicina, Departamento de Radiologia, São Paulo SP, Brasil.

Correspondence: Daphine C. Grassi; Departamento de Radiologia, Divisão de Ressonância Magnética da HCFMUSP; Av. Dr. Enéas de Carvalho Aguiar, 255; 05403-900 São Paulo SP, Brasil; E-mail: daphinecgrassi@gmail.com

Support: Grants 2015/18136-1, 2016/05547-6 and 2017/17065-9 from São Paulo Research Foundation - FAPESP.

Conflict of interest: There is no conflict of interest to declare.

Received 17 October 2017; Received in final form 29 November 2017; Accepted 12 December 2017. 
One of the most important aspects for guaranteeing an optimized approach for treating a TBI victim is understanding how the trauma occurred and providing a detailed and prompt clinical examination of the victim. Neurotrauma mechanisms are complex, and multiple types of injuries over the CNS may coexist, such as skull fractures, contusions, hematomas, and diffuse axonal injury ${ }^{3,4}$. The Glasgow Coma Scale (GCS) is the most widely used clinical classification system and depends on the best eye, verbal and motor responses. According to the GCS, a TBI can be graded as mild, moderate or severe. This scoring system was created in 1975 and lacks the sensitivity to predict subtle but meaningful residual dysfunctions, such as physical, cognitive, psychological and behavioral deficits ${ }^{5}$.

More comprehensive clinical algorithms have been developed to determine the mortality and persistent disabilities in TBI victims. Two of the most used and well-known are the IMPACT and CRASH algorithms. The IMPACT algorithm was developed during clinical trials of severe TBI victims, and the CRASH algorithm was developed with mild and moderate TBI patients. Both algorithms use clinical predictors such as age, GCS score and pupillary reactivity at hospital admission. The addition of biomarkers, such as protein S100-beta, microtubule associated protein 2 and myelin basic protein, as well as imaging data from head computed tomography (CT) scans, has been shown to improve the reliability of these prognostic models ${ }^{5}$. However, the clinical manifestations present after traumatic events vary, and predicting prognosis in an individual patient remains challenging in daily practice p.,67. $^{5}$.

In particular, patients with diffuse axonal injury frequently exhibit an apparent discrepancy between clinical status (usually moderately to severely compromised) and early imaging findings (often normal or minimally abnormal) ${ }^{3,4}$. Moreover, it is not well established why some survivors regain complete function while others remain severely disabled. Therefore, it is critical to understand the pathophysiology of diffuse axonal injury and to foster the development of noninvasive neuroimaging tools to reveal the damage to the central nervous system and provide guidance for therapeutic decisions and counseling for TBI patients and their families.

\section{PATHOPHYSIOLOGY OF DIFFUSE AXONAL INJURY}

Diffuse axonal injury is defined as wide axonal injury with microscopic and macroscopic components, which may only be visible in severe cases upon CT and magnetic resonance imaging (MRI). The mechanism of injury is based on the inertia of the brain: when fast and strong accelerations and decelerations occur, different structures with distinct densities (such as gray and white matter) suffer shearing and straining forces that stretch and damage axons, leading to diffuse axonal injury ${ }^{2,4}$. Commonly affected brain sites include the corpus callosum, fornices, subcortical white matter and cerebellum ${ }^{7}$. Once the victim presents in a comatose state with a GCS less than 8, the probability of brainstem involvement becomes significant and the prognosis worsens ${ }^{8}$.

It is very important to understand that diffuse axonal injury does not only consist of the axonal injury itself. Complex neuropathological processes ensue, such as an inflammatory response secondary to the traumatic event associated with protein deposition. After direct axonal injury, multiple changes occur microscopically, including cellular death, synaptic dysfunction, activation of glial cells and anomalous protein deposition (Tau and A $\beta$ proteins) ${ }^{9,10,11}$.

Damage to an axon does not always lead to disconnection: connections may occasionally still be present, but it is not well known if they remain functional ${ }^{12}$. It is well established that neuronal death per se evolves into Wallerian degeneration, which is defined as the progressive anterograde disintegration of axons and accompanying demyelination that occurs after injury to the proximal axon or cell body ${ }^{13}$. Furthermore, it has been increasingly recognized that anomalous protein deposition secondary to axonal injury might be related to the future manifestation of Alzheimer's disease in some patients ${ }^{14,15}$.

Histopathologically, diffuse axonal injury can be divided into three degrees: grade I: microscopically widespread axonal injury in any location; grade II: grade I findings plus focal lesions in the corpus callosum; and grade III: grade II findings plus focal lesions in the rostral portion of the brainstem ${ }^{16}$.

\section{NEUROIMAGING}

Neuroimaging evaluation of a TBI patient is based on CT and MRI studies. During emergency care, TBI victims are usually first evaluated with CT scans, which are fast and accurate for identifying life-threatening conditions that may require prompt intervention, such as extra-axial hematomas ${ }^{7}$.

There is a mismatch between the CT findings and clinical presentation in TBI patients. For instance, punctate microhemorrhages on the corpus callosum and gray-white matter junction are shown in only $10 \%$ of all TBI patients. Within two weeks after a traumatic event, neuronal loss can be inferred on CT as a discrete ventricular enlargement in some patients ${ }^{7,14}$.

An MRI, despite being less widely available and requiring longer scan times than a CT, is the best modality to assess brain injuries because it provides a better identification of anatomic features and higher spatial resolution ${ }^{3}$. Hemorrhages are represented by a loss of signal in gradient echo and susceptibility-weighted sequences (Figure 1), whereas areas of edema present as high signals in T2-weighted and fluid attenuation inversion recovery (FLAIR) sequences (Figure 2).

Nevertheless, more advanced techniques, such as diffuse tensor imaging (DTI), are more sensitive to neuronal lesions in areas that appear normal on conventional MRI sequences, especially in patients with diffuse axonal injury ${ }^{14,17,18,19}$. 

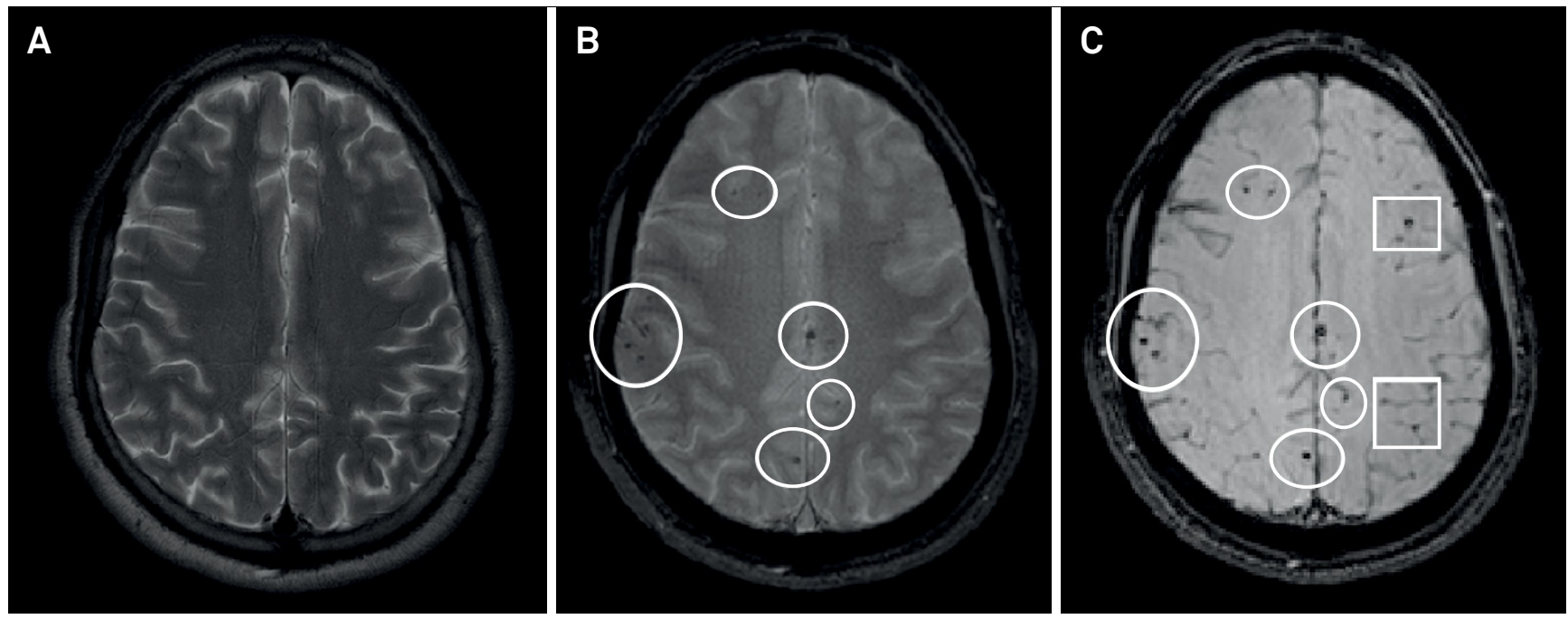

Figure 1. A 19-year-old male patient with diffuse axonal injury and Glasgow Coma Scale score of 8 after a motorcycle accident. While T2 conventional sequences (A) are rather insensitive for hemorrhagic lesions, T2*-GRE (B) shows numerous foci of signal loss (circles) in the subcortical white matter corresponding to areas of extravascular blood. Susceptibility weighted imaging (C) is even more sensitive than the previous two sequences, exhibiting more conspicuous (circles) and numerous lesions (squares) on both cerebral hemispheres. The images were performed sequentially during the same examination at a 3 Tesla scanner.
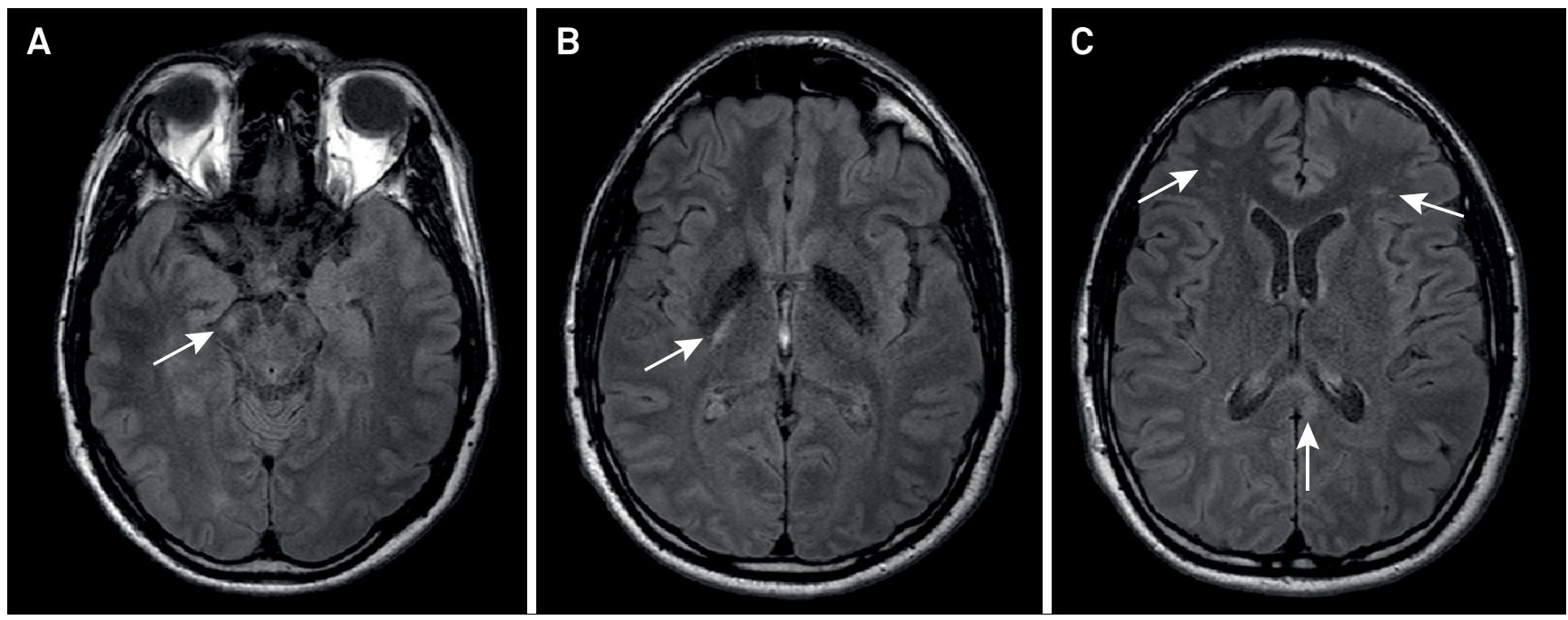

Figure 2. A 27-year-old male patient with moderate TBI due to a motorcycle accident. At admission, his Glasgow Coma Scale score was 10 and he presented with left hemiparesis and left third nerve palsy. Axial FLAIR images show hyperintense lesions (arrows) in the lateral aspect of the right cerebral peduncle (A), right internal capsule (B), splenium of the corpus callosum, as well as subtle lesions in the subcortical bilateral frontal white matter (C).

\section{DIFFUSION WEIGHTED IMAGING}

Since its medical debut, diffusion weighted imaging (DWI) has largely been used in routine clinical practice in applications ranging from diagnosing stroke to helping physicians determine tumor cellularity. Diffusion weighted imaging is an integral part of any MRI brain examination and is of paramount importance in radiological diagnosis.

Diffusion weighted imaging is based on the random motion of water molecules, which was first described by the botanist Robert Brown in 1827. In a homogeneous liquid environment without any barriers, water diffusion is free (isotropic); in other words, it has no preferred direction. In contrast, when there are surrounding structures restricting water molecule movement, a preferred diffusion direction becomes apparent (anisotropic diffusion) ${ }^{20}$.

In the brain, several components hinder water diffusion in all directions, making the diffusion anisotropic. This anisotropic diffusion depends on the geometry and composition of natural barriers, such as cell membranes, myelin sheaths and primary microstructural components (neurofilaments and microtubules). For this reason, anisotropy is markedly high in well-organized white matter tracts and is lower in cerebrospinal fluids ${ }^{21}$. 
Diffusion weighted imaging shows hyperintensities in pathological conditions, such as in areas of high cellularity density (tumors), abnormal cellular uptake of water (cytotoxic edema) or entrapment of water between myelin membranes (intramyelinic edema). However, other increases in water concentration, such as observed in vasogenic edema or gliosis, also cause nonspecific hyperintensity on DWI, which is a T2-weighted sequence ${ }^{22}$.

To distinguish between true restriction of water molecule diffusion and artifacts (T2 shine-through effects), apparent diffusion coefficient (ADC) maps were developed. When analyzing each voxel from the image with two different $b$ values, it is possible to quantify water diffusivity and convert this information into a visual map (ADC map). The term "apparent" reflects the fact that calculated ADCs in tissues vary according to the previously attributed $b$ values. Moreover, the ADC information corresponds to each voxel (in the order of millimeters) but not at the microscopic level of cell structures (in the order of a few micrometers). On ADC maps, restricted water diffusion is confirmed as a low signal intensity ${ }^{23,24}$.

In patients with traumatic injury, cytotoxic edema usually occurs in cases of cortical contusion and diffuse axonal injury. Cortical contusions usually affect both superficial cortical gray matter and subcortical white matter but do not typically follow a vascular distribution. In acute diffuse axonal injury, it is possible to detect multifocal areas with restricted diffusion, which appear bright on DWI and dark on ADC maps. Predilection sites are the corpus callosum, especially the splenium, cerebral peduncles, deep white matter structures and gray-white matter interface (Figure 3). This restricted water diffusion may vanish in a few days or may evolve into residual lesions with a persistent high signal intensity on FLAIR and T2-weighted images ${ }^{25}$.

\section{DIFFUSION TENSOR IMAGING}

The tensor model was proposed to characterize and quantify diffusion anisotropy. By measuring diffusion in at least six different gradient directions applied to the three different axes (X - horizontal, Y - vertical and Z - perpendicular to $\mathrm{X}$ ), it is possible to determine an average water diffusion according to the distance and intensity for each voxel ${ }^{26,27}$.

Fractional anisotropy (FA) is a scalar measure that reflects microstructural geometry and is very high (close to 1) in normal white matter but is usually lower in damaged white matter and is close to zero in the cerebrospinal fluid ${ }^{27}$. Colormaps can be created based on the first eigenvector that composes the FA calculation in which each color represents the main diffusion direction of white matter tracts (conventionally, red is used for left-right, green for anteroposterior, and blue for superior-inferior directions), and the degree of brightness is proportional to the magnitude of anisotropy ${ }^{28,29}$ (Figure 4).

The mean diffusivity reflects the overall degree of water diffusion in all directions, regardless of its orientation dependence $^{30}$. Radial and axial diffusivity are other quantitative DTI parameters. Axial diffusivity is the principal tensor direction (first eigenvector) with its associated magnitude (first eigenvalue), and radial diffusivity represents the other two directions perpendicular to the principal direction of the diffusion tensor. Investigations with animal models have indicated that radial diffusivity correlates with demyelination, whereas axial diffusivity seems to be related to more profound tissue damage and axonal loss ${ }^{31,32}$. Still, translational characteristics of these studies remain to be proven.

Several acquisition parameters influence the quality of diffusion data. The ability to obtain a valuable dataset is strongly related to the strength of the gradient coils and the maximum $b$ values that can be achieved. For most brain studies, a $b$ value
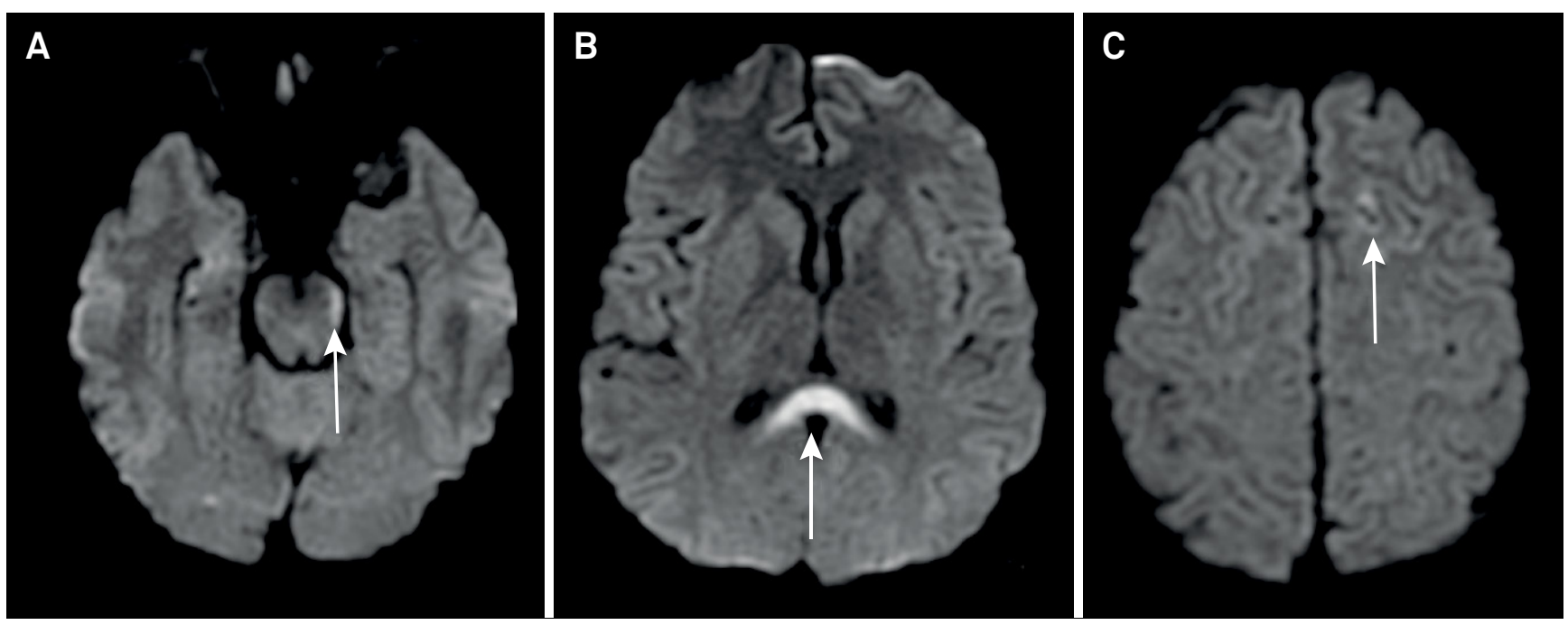

Figure 3. Diffusion-weighted images in the axial plane of a young patient four days after a head injury demonstrate bright lesions (arrows) in areas typically affected in diffuse axonal injury: left cerebral peduncle (A), splenium of the corpus callosum (B), and subcortical frontal white matter (C). The lesions were dark on ADC maps (not shown). 
of $1000 \mathrm{~s} / \mathrm{mm}^{2}$ is usually adequate and results in an effective compromise between sensitivity and the signal-to-noise ratio. Ideally, images should be acquired with high isotropic spatial resolution (1-2 $\left.\mathrm{mm}^{3}\right)$. Many gradient directions, optimally at least 32, should be applied. However, there is a trade-off between the optimal acquisition parameters and the scanning time. Head motion should be minimized as much as possible. Preprocessing steps may, however, partly attenuate motion artifacts and the eddy current artifacts ${ }^{33,34}$.

Distinct methods are available to analyze diffusion tensor images. Herein, we aim to briefly discuss the basic principles, advantages and caveats of each main DTI analytical method, namely, region-of-interest analysis, tractography and voxelwise analysis, along with the relevant findings of recent DTI studies of TBI patients.

\section{REGION-OF-INTEREST ANALYSIS}

In region-of-interest (ROI) analysis, diffusion parameters are obtained from a predetermined area of the brain or around a specific anatomic structure. The area-of-interest can be manually drawn, and there is no requirement to use an anatomic atlas. This method is also suitable for studies in patients with large brain lesions in different sites or great anatomic distortions that could bias other analytical methods that require registration steps. Once the ROI is determined, the mean values of the water diffusion parameters are obtained $^{33,34}$ (Figure 5).

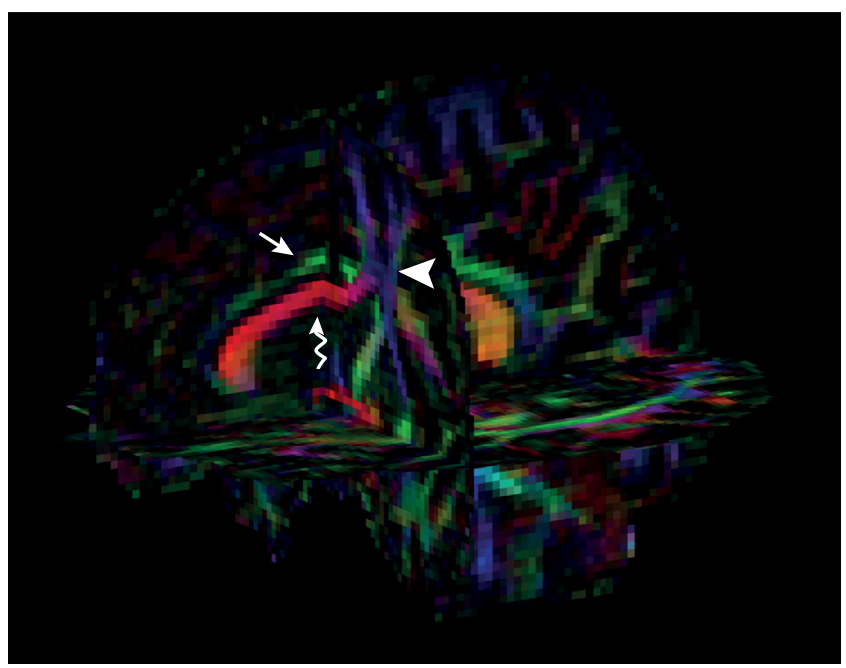

Figure 4. Three-dimensional FA colormap of an 18-yearold TBI victim. The patient was admitted with a Glasgow Coma Scale score of 8 after a motorcycle accident, despite the use of a helmet. Conventionally, long association fibers with anteroposterior direction such as the cingulum are represented in green (straight arrow), inter-hemispheric commissural fibers (e.g., corpus callosum, curved arrow) with left-right direction are represented in red, while projection tracts with superior-inferior route such as the corticospinal tract are represented in blue (arrowhead).
One advantage of this method is that even small brain regions, such as subcortical areas and deep basal ganglia, can be assessed. Another benefit is that multiple comparison errors are less prominent if the investigator predefines an a priori hypothesis and fewer areas are examined. Region-of-interest analysis identifies even subtle changes and is thus one of the most sensible and straightforward analytical methods available ${ }^{33}$.

However, certain considerations for ROI analysis must be underscored: some information may be missed when only one area is being studied; it may be difficult to compare the same ROIs among individuals due to intrinsic variability; and the process of delimitation of the ROI can be laborious if it appears in multislice images or in the case of big data with multisubject comparisons. It is extremely important to know where to look for the changes; ROI analysis of nonaffected areas can identify normal parameters, but truly compromised areas can be missed. The manual nature of the ROI drawing may lead to the low reproducibility of intra- and intersubject ROI correspondence; hence, anatomic atlas tools or semi-automatic methods are advisable. For this reason, it is possible to standardize ROI studies by using segmentation and registration steps; segmentation helps determine the area of interest, whereas registration matches the corresponding points among all images in different subjects ${ }^{33,34,35}$.

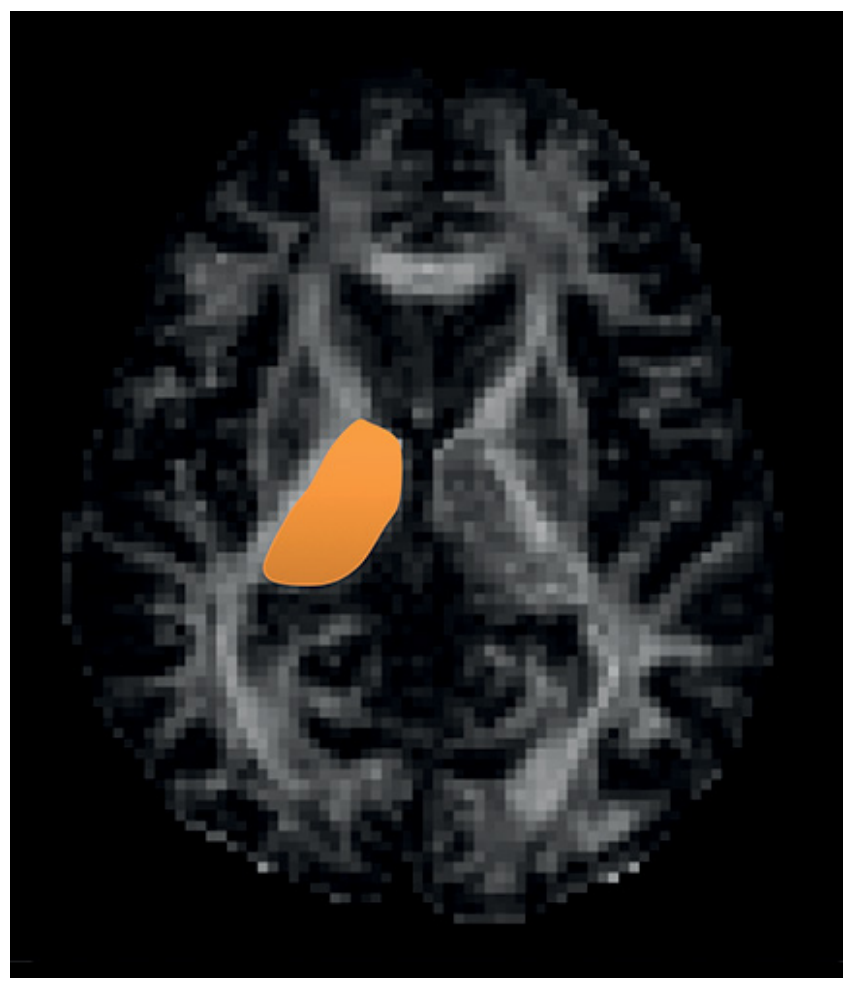

Figure 5. Diffusion parameters can be extracted from a selected ROI. In this case of a 42-year-old woman who suffered physical aggression and presented with severe TBI, the right thalamus (orange shape) was manually delineated in the FA map. 
Mac Donald et al. ${ }^{36}$ demonstrated that DTI could detect white matter injury in a mouse model of diffuse axonal injury and could determine the approximate timing of injury. These authors extracted diffusion parameters with ROI analyses from the corpus callosum and the external capsule and compared the findings with histological and electron microscopy results. In comparison with uninjured mice, anisotropy measures were lower in the injured group at all stages. During the early acute phase (less than one day), axial diffusivity was reduced and axonal injury was present histologically. During the subacute phase (one week to one month after injury), a reduction of anisotropy was accompanied by increases in axial diffusivity, radial diffusivity and mean diffusivity, which reflected the dominance of demyelination and edema at histological evaluation. The authors proposed that if similar mechanics are present in human TBI, these DTI changes could be used for novel clinical and forensic applications. However, the authors did not find any correlation between the severity of histological damage and the DTI parameters ${ }^{36}$.

Another study evaluated 10 healthy individuals and five patients with mild TBI within 24 hours of injury and one month later. The authors extracted diffusion parameters with ROIs positioned in the corpus callosum and internal and external capsules. Soon after the injury, TBI patients demonstrated significant reductions in anisotropy compared with controls. In the 30-day-control, two patients showed slight increases in FA values when compared with their initial results, which was considered a possible sign of recovery ${ }^{37}$.

According to Huisman et al. ${ }^{18}$, changes in water diffusion anisotropy do occur in TBI, and these changes may be biomarkers for severity of tissue injury and predictors for outcome. Patients were analyzed within seven days of the event, and the data were compared with those from a control (healthy) group. The studied regions included the internal capsule, splenium, thalamus and putamen. This study showed that FA was significantly decreased in the posterior limb of the internal capsule and splenium of the corpus callosum. Furthermore, there was a statistically significant correlation between the FA values in the diffuse axonal injury predilection sites and the severity of head injury, as measured by acute and subacute neurologic assessments (acute GCS and Rankin scores $)^{18}$.

In addition, another longitudinal study analyzed 11 TBI victims in acute (less than seven days) and subacute (from eight days to rehabilitation discharge) stages and correlated DTI findings with a disability rating scale. Eleven ROIs were chosen based on previous studies that demonstrated an association between FA and functional or cognitive outcomes in TBI. It was apparent that the FA values varied according to the pathophysiologic processes of TBI. During the acute phase, the FA values were lower than they were in the subacute stage, possibly because of brain edema. Consequently, subacute values were more likely to reflect the axonal structural integrity. Therefore, these authors suggested that the optimal timing of DTI data acquisition for TBI prognostication might be during the subacute stage of injury ${ }^{38}$.

\section{TRACTOGRAPHY}

Three-dimensional visualization of DTI information is also possible. Tensor information allows the trajectories to be estimated by inferring white matter fiber orientations. Tractography allows the parcellation of white matter, and this information may be particularly useful in anatomofunctional studies because white matter bundles are linked to specific cognitive, language, behavioral, and motor functions (Figure 6) ${ }^{39}$.
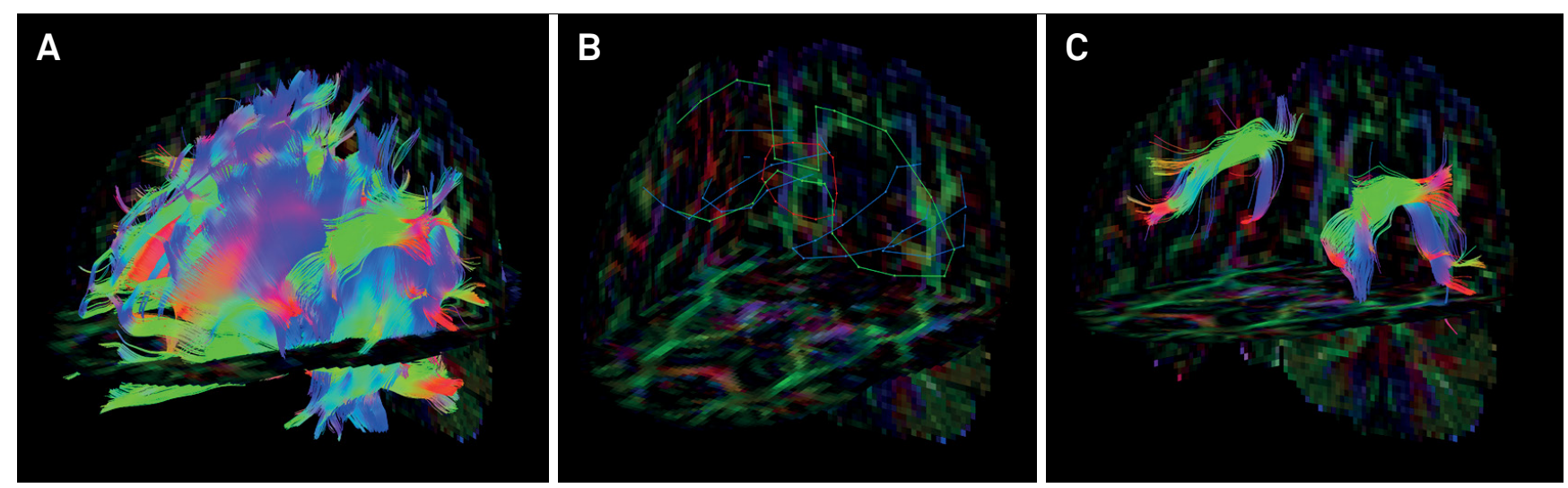

Figure 6. A young female patient had a motorcycle accident and presented at the emergency room with a Glasgow Coma Score of 3. She persisted with language impairment one year after the traumatic event. It is possible to evaluate DTI parameters from any tract related to a specific cognitive domain. The superior longitudinal fasciculus is linked to language skills because it interconnects Broca's area (responsible for speech production in the frontal lobe), Geschwind's area (semantic processing in the parietal lobe) and Wernicke's territory (speech comprehension in the temporal lobe). First, whole-brain tractography was obtained with a brute-force approach from the full tensor data (A). A set of "AND", "SEED" and "NOT" ROls was placed on both cerebral hemispheres based upon a priori anatomical knowledge (B). The superior longitudinal fasciculi were then virtually dissected with a deterministic streamline approach and displayed on a FA color-encoded map (C). 
Tractography consists of three processing steps: seeding, propagation and termination. Seeding involves determining the area from which the fiber bundles will be drawn, and the most common approach is to define an ROI and placing one or more seeds in the expected trajectory of the tract. Another possible method is to use automatic seeding for the whole brain from a full set of tensor data ${ }^{29,39}$.

Propagation is how visual schemes of fibers are generated, and different algorithms are developed to link intervoxel information. Algorithms are based on deterministic or probabilistic approaches. The first approach is based on a streamline principle: from the seed location (ROI) nearby voxels will be linked to the same streamline if their principal eigenvectors have congruous orientations or similar FA values. Probabilistic tractography, in contrast, represents an estimation based on multiple possible fiber directions in each seed. For this reason, probabilistic tractography tends to disperse trajectories more than deterministic methods and has the potential to delineate a greater proportion of the white matter tract ${ }^{28,29,39,40}$.

Finally, termination is the last step of the fiber tracking procedure with well-defined criteria. Typically, FA thresholds (usually higher than 0.25 to avoid contamination by CSF and grey matter) and turning angle thresholds (generally $30^{\circ}$ or $60^{\circ}$ depending on the known curvature of the tract of interest) are used. This method of "virtual dissection" allows the isolation of specific anatomic fiber pathways from DTI datasets and has been proven useful in several adult and pediatric conditions (Figure 7) ${ }^{39}$.

Nevertheless, there are some important shortcomings that must be taken into account when analyzing tractography. This method estimates fiber tract anatomy on a macro scale. Voxels are in the order of millimeters, whereas the axonal diameter is in the order of microns. It is also not possible to differentiate afferent from efferent bundles. Moreover, the assumption of homogeneous unidirectional tensors is unrealistic because many brain regions comprise more than one fiber bundle. Crossing, diverging or kissing fibers result in incorrect direction estimations and pathways and may lead to abrupt tract termination. Diffusion spectrum imaging, q-space imaging, q-ball imaging, and high angular resolution diffusion imaging are more sophisticated approaches that may overcome some of these limitations ${ }^{40}$.

Wang et al. $^{41}$ studied 12 patients who suffered from severe TBIs within seven days and nine months after injury. They analyzed the corpus callosum, fornix and peduncular projections with deterministic tractography. The authors could identify at least one DTI parameter that demonstrated diffuse axonal injury-associated alterations in each region. Furthermore, they demonstrated a good correlation between the DTI findings and long-term prediction outcome, as measured with Glasgow Outcome Scale-Extended scores ${ }^{41}$.

Another group demonstrated that patients with mild TBI have reduced FA values in various white matter locations and various fiber bundles within 5.5 months after trauma. In comparison with healthy-matched controls, the authors demonstrated lower anisotropy in multiple white matter regions, predominantly in the cerebral lobar white matter, cingulum and corpus callosum, using deterministic tractography. A minority of fibers showed premature discontinuation on fiber tracking, and the authors presumed that this may have been caused by the presence of sharply angulated fibers or by small areas of hemosiderin that were not visible on $\mathrm{MRI}^{42}$.
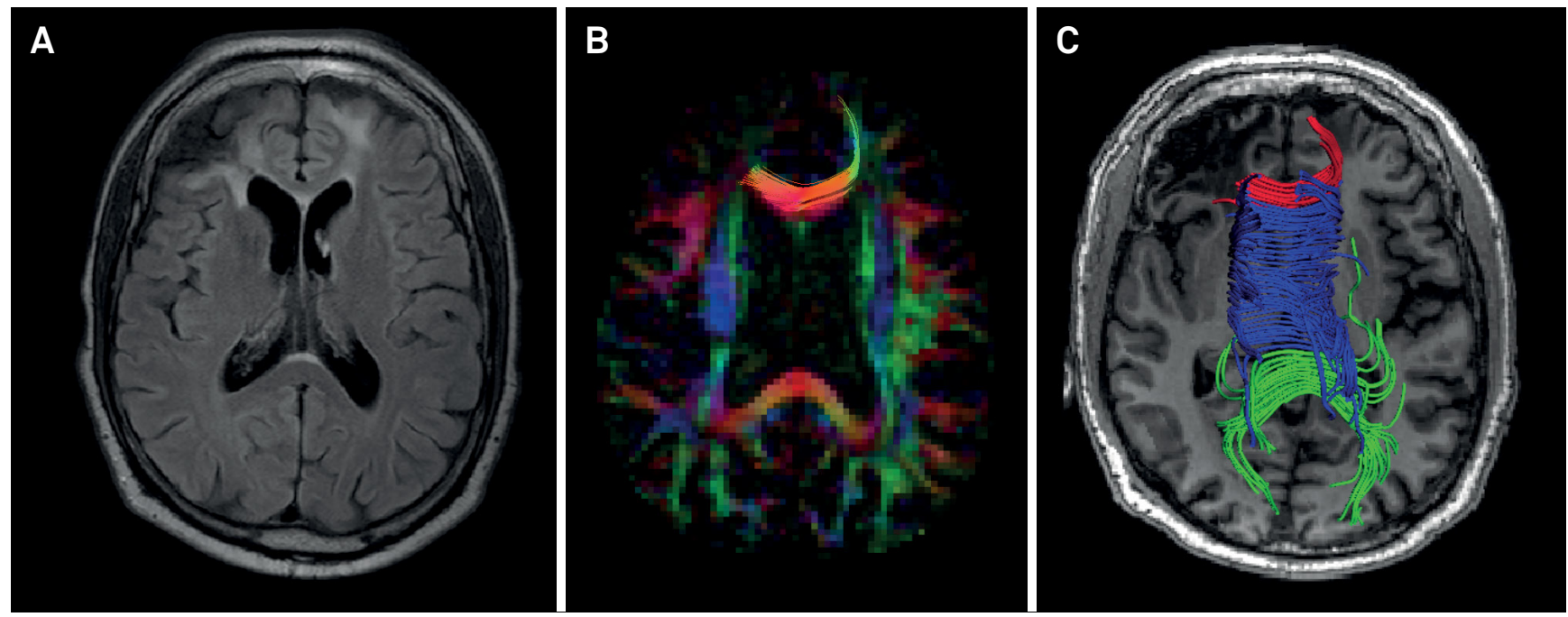

Figure 7. Axial FLAIR image of an adult male patient with chronic post-traumatic sequelae shows gliosis in the frontal lobes, mainly in the right side (A). FA colormap demonstrates paucity of white matter fibers and reduction of brightness due to reduction of FA values in the frontal lobes, more pronounced in the right side (B). DTI-based tractography of the splenium, body and genu of the corpus callosum (represented in green, blue, and red colors, respectively) shows premature termination of streamlines of peripheral fibers of the genu projecting to or from the prefrontal regions coincidental to the areas of low signal intensity on the axial T1-weighted image (C). 
A large study evaluated 106 chronic TBI patients who had no abnormalities on conventional MRI in comparison with 62 healthy controls. The investigators applied a deterministic approach to extract the volume and FA measures of long association tracts from the uncinated fasciculus, superior cingulum, temporal cingulum, superior longitudinal fasciculus, arcuate fasciculus, inferior fronto-occipital fasciculus and inferior longitudinal fasciculus. Injured patients demonstrated reduced FA values in both uncinated fasciculi, both inferior fronto-occipital fasciculi and in the right inferior longitudinal fasciculus. However, the tract volumes were not significantly decreased in injured patients ${ }^{43}$.

A recent study evaluated trauma-exposed police officers with and without post-traumatic stress disorder using a 3T system. The authors applied an automated and unbiased reconstruction of white matter tracts using a global probabilistic tractography method known as TRACULA (TRActs Constrained by UnderLying Anatomy) ${ }^{44}$ and found significantly higher mean diffusivity in the right uncinate fasciculus in the affected group. The uncinate fasciculus is the major white matter tract that connects the amygdala to the prefrontal cortex. These authors also found that the mean diffusivity of the right uncinate fasciculus was positively associated with anxiety symptoms in patients with post-traumatic stress disorder ${ }^{45}$.

Head injury survivors usually present with persistent cognitive symptoms that impair their quality of life, such as deficits in language performance and executive function. In the future, tractography studies could be used to monitor the benefits of target therapies in these patients by evaluating DTI metrics in specific white matter tracts linked to specific functional domains.

\section{VOXELWISE ANALYSIS}

Voxelwise analysis has become more popular because its automatic approach requires minimal intervention and less user dependence. Voxelwise analysis is suitable for global analyses of brain parenchyma and is particularly useful for large group comparisons of individuals with no significant distortions in brain anatomy ${ }^{33,46}$.

Primarily, the images must be standardized into a template to ensure that each voxel corresponds to the same anatomic location in all subjects. Thus, a critical step is to define the best way to register and compare multiple images from different individuals in an accurate manner ${ }^{33}$.

Previously, voxelwise analyses were performed with voxelbased morphometry using T1 weighted-images. Although some constraints related to image registration, segmentation and smoothing are present, voxel-based morphometry can still be used to explore diffusion images in particular research scenarios.

Tract-based spatial statistics is the currentleading method for voxelwise analysis using nonlinear image transformation of FA images across subjects ${ }^{46}$. Tract-based spatial statistics uses the mean FA values from individual subjects to create a skeletonized map with the local maximal FA values for each tract. With this approach, the differences in all the voxels of the brain, except those from the skeleton voxels are ignored. Another challenge is registration inaccuracies of areas with high contrast FA values, such as those areas adjacent to ventricles ${ }^{33,47}$. A more recent alternative approach for the registration step of tract-based spatial statistics is to use the full tensor information with a complementary tool, known as the DTI-ToolKit, which has been shown to reduce the number of misassigned voxels by a total of seven ${ }^{47,48}$.

In voxelwise analysis, all voxels in the image are compared with each other in a local manner, and hence statistical procedures to control for multiple comparison errors might be carried out. These may, in turn, reduce the sensitivity for more subtle findings in particular regions ${ }^{33}$.

Our group evaluated 20 adults with moderate to severe TBI using a 3.0T MRI scanner during the acute ( $\mathrm{t}_{1}<3$ months), subacute $\left(6<\mathrm{t}_{2}<9\right.$ months) and chronic stages $\left(12<\mathrm{t}_{3}<15\right.$ months $)$ following trauma. According to tract-based spatial statistics analysis, the patients exhibited one large cluster with statistically significant lower FA values $(\mathrm{p}<0.001)$ at all times $\left(t_{1}, t_{2}\right.$, $t_{3}$ ) compared with the controls (Figure 8 ), but the number of affected voxels decreased over time by $2 \%$ at $t_{2}$ and by $7.3 \%$ at $t_{3}$. During the chronic stage $\left(t_{3}\right)$, patients recovered from white matter damage in comparison with the acute stage $\left(t_{1}\right)$ with significant increases in FA in the bilateral anterior thalamic radiations, forceps major and minor, corticospinal tracts, cingulum, uncinate, inferior fronto-occipital, superior and inferior longitudinal fasciculi. Patient performance on cognitive measures was suboptimal at all three stages but also improved over time in the same fashion as white matter recovery ${ }^{49}$.

One study applied voxel-based analysis and ROI analysis in 10 adolescent patients with mild TBI who were assessed within one week after injury and compared with a pairedcontrol group. The results indicated increased FA, decreased radial diffusivity and unchanged axial diffusivity in multiple brain regions, which may be related to axonal cytotoxic edema and reflect acute injury. Moreover, the alterations in DTI metrics were highly correlated with postconcussive symptoms severity and emotional distress ${ }^{50}$.

Lipton et al..$^{51}$ retrospectively analyzed 17 cognitivelyimpaired mild TBI victims who underwent neuroimaging studies between eight months and three years after the trauma event and compared these patients with a healthy cohort of 10 individuals. Voxel-based analysis showed multiple areas of lower FA and high mean diffusivity in the white matter bilaterally, especially in the corpus callosum, subcortical white matter and internal capsules ${ }^{51}$.

Another study indicated extensive changes in major intra- and interhemispheric white matter tracts in patients with diffuse axonal injury. The authors carried out both ROI analysis and voxel-based analysis in nine chronic TBI patients 

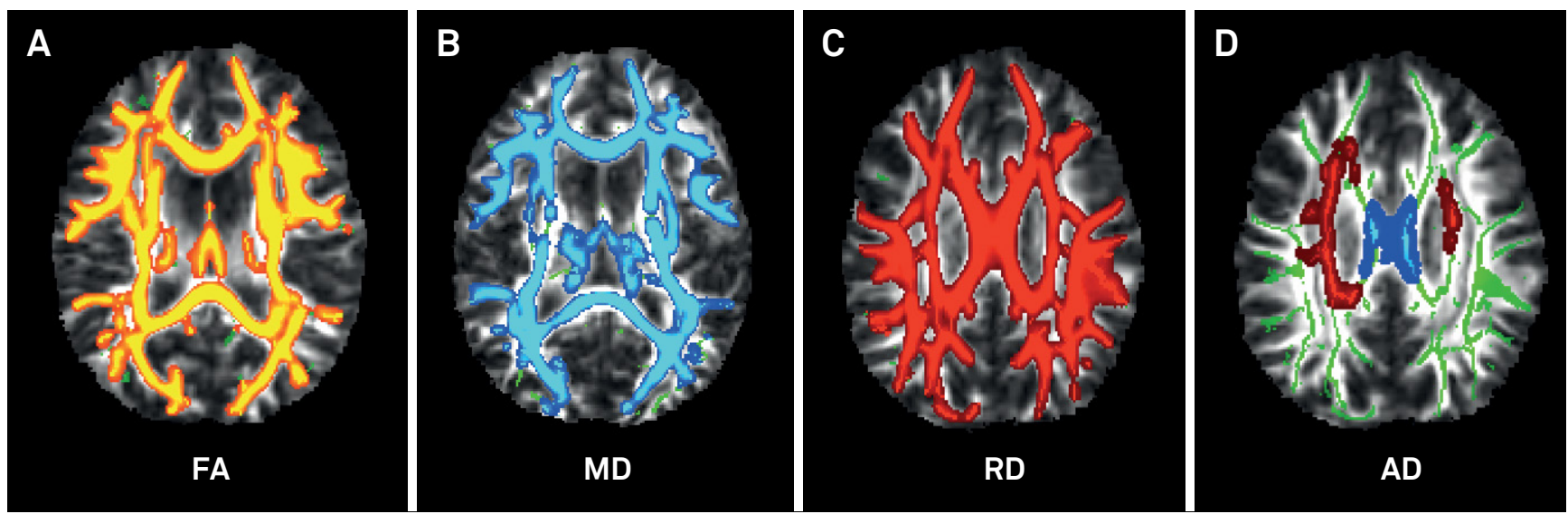

Figure 8. Twenty patients with acute moderate-to-severe TBI were compared with 20 age- and sex-matched controls with tractbased spatial statistics. The patients exhibited one large cluster with statistically significant lower FA values (A). The TBI patients also demonstrated significant increases in mean diffusivity (B) and radial diffusivity (C) in extensive areas of the brain, as well as increased acute diffusivity in a less extensive area (shown in red), except for the corpus callosum, which showed increased acute diffusivity (depicted in blue) (D). All comparisons were statistically significant.

(approximately four years after the event) and in 11 healthy individuals. The results indicated significantly lower FA values in the corpus callosum, internal and external capsules, superior and inferior longitudinal fascicles and in the fornix in the TBI group. Furthermore, ADC values were increased not only where the FA values were lower but also in otherwise-normal regions, possibly indicating that $\mathrm{ADC}$ may be an even more sensitive measurement than FA for detecting widespread white matter damage ${ }^{52}$.

\section{CONCLUSION}

Traumatic brain injury remains a major public health problem worldwide. Computed tomography and MRI have played a crucial role in the acute setting, but several challenges arise when applying neuroimaging methods to predict clinical outcomes in patients with a broad range of injury severity, especially in individuals who develop persistent symptoms despite minor findings on standard imaging.

Diffuse tensor imaging takes advantage of the intrinsic property of anisotropic diffusion of water molecules in brain tissues to probe tissue integrity and organization. Although increasing publications have reported applying this technique in various clinical and research scenarios, DTI principles and its various methodological approaches remain unfamiliar to some allied-health professionals. We reviewed some advantages and shortcomings of the most commonly applied analytical methods (ROI, tractography and voxelwise analyses) along with their applications in the investigation of TBI.

Particularly in patients with diffuse axonal injury, natural barriers to free water diffusion, such as the cytoskeleton, axons and myelin sheaths, may be damaged, leading to the ubiquitous finding of reduced FA values in distinct brain areas. Increased radial diffusivity and increased mean diffusivity usually accompany these FA changes. It seems that abnormalities in DTI metrics may correlate with the timing of head injury, severity biomarkers and long-term prognosis. In the future, DWI and DTI may also aid in the selection of TBI patients for targeted therapies and in monitoring the effectiveness of treatments.

Upcoming investigations should attempt to select more homogeneous groups of patients and clearly state inclusion and exclusion criteria. Longitudinal studies with a combined quantification of DTI metrics, instead of transversal studies with putative evaluation of isolated indices, should enhance comprehension of TBI pathophysiology. These studies might foster the goal of alleviating the burden associated with TBI.

\section{References}

1. Corso P, Finkelstein E, Miller T, Fiebelkorn I, Zaloshnja E. Incidence and lifetime costs of injuries in the United States. Inj Prev. 2006;12(4):212-8. https://doi.org/10.1136/ip.2005.010983

2. Greve MW, Zink BJ. Pathophysiology of traumatic brain injury. Mt Sinai J Med. 2009;76(2):97-104. https://doi.org/10.1002/msj.20104

3. Morais DF, Spotti AR, Tognola WA, Gaia FF, Andrade AF. Clinical application of magnetic resonance in acute traumatic brain injury. Arq Neuropsiquiatr. 2008 Mar;66(1):53-8. https://doi.org/10.1590/S0004-282X2008000100013
4. Andrade AF, Paiva WS, Amorim RL, Figueiredo EG,

Rusafa Neto E, Teixeira MJ. [The pathophysiological mechanisms following traumatic brain injury].

Rev Assoc Med Bras (1992). 2009

Jan-Feb;55(1):75-81. Portuguese.

https://doi.org/10.1590/S0104-42302009000100020

5. Sandsmark DK. Clinical outcomes after traumatic brain injury. Curr Neurol Neurosci Rep. 2016;16(6):52. https://doi.org/10.1007/s11910-016-0654-5. 
6. Vieira RC, Paiva WS, Oliveira DV, Teixeira MJ, Andrade AF, Sousa RM. Diffuse axonal injury: epidemiology, outcome and associated risk factors. Front Neurol. 2016 Oct;7:178. https://doi.org/10.3389/fneur.2016.00178

7. Provenzale JM. Imaging of traumatic brain injury: a review of the recent medical literature. AJR Am J Roentgenol. 2010;194(1):16-19. https://doi.org/10.2214/AJR.09.3687

8. Skandsen T, Kvistad KA, Solheim O, Strand IH,

Folvik M, Vik A. Prevalence and impact of diffuse axonal injury in patients with moderate and severe head injury: a cohort study of early magnetic resonance imaging findings and 1-year outcome. J Neurosurg. 2010;113(3):556-563. https://doi.org/10.3171/2009.9.JNS09626

9. Li J, Li XY, Feng DF, Pan DC. Biomarkers associated with diffuse traumatic axonal injury: exploring pathogenesis, early diagnosis, and prognosis. J Trauma. 2010 Dec;69(6):1610-8. https://doi.org/10.1097/TA.0b013e3181f5a9ed

10. Olsson A, Csajbok L, Ost M, Höglund K, Nylén K, Rosengren L et al. Marked increase of beta-amyloid(1-42) and amyloid precursor protein in ventricular cerebrospinal fluid after severe traumatic brain injury. J Neurol. 2004 Jul;251(7):870-6. https://doi.org/10.1007/s00415-004-0451-y

11. Magnoni S, Esparza TJ, Conte V, Carbonara M, Carrabba G, Holtzman DM et al. Tau elevations in the brain extracellular space correlate with reduced amyloid- $\beta$ levels and predict adverse clinical outcomes after severe traumatic brain injury. Brain. 2012 Apr;135(Pt 4):1268-80. https://doi.org/10.1093/brain/awr286

12. Büki A, Povlishock JT. All roads lead to disconnection? Traumatic axonal injury revisited. Acta Neurochir (Wien). 2006 Feb;148(2):181-93. https://doi.org/10.1007/s00701-005-0674-4

13. Chen YJ, Nabavizadeh SA, Vossough A, Kumar S, Loevner LA, Mohan S. Wallerian degeneration beyond the corticospinal tracts: conventional and advanced MRI findings. J Neuroimaging. 2017 May;27(3):272-80. https://doi.org/10.1111/jon.12404

14. Meythaler JM, Peduzzi JD, Eleftheriou E, Novack TA. Current concepts: diffuse axonal injury-associated traumatic brain injury. Arch Phys Med Rehabil. 2001;82(10):1461-1471. https://doi.org/10.1053/apmr.2001.25137

15. Magnoni S, Brody DL. New perspectives on amyloid-beta dynamics after acute brain injury moving between experimental approaches and studies in the human brain. Arch Neurol. 2010;67(9):1068-1073. https://doi.org/10.1001/archneurol.2010.214

16. Adams JH, Doyle D, Ford I, Gennarelli TA, Graham DI, McLellan DR. Diffuse axonal injury in head-injury - definition, diagnosis and grading. Histopathology. 1989;15(1):49-59. https://doi.org/10.1111/j.1365-2559.1989.tb03040.x

17. Shenton ME, Hamoda HM, Schneiderman JS, Bouix S, Pasternak $\mathrm{O}$, Rathi $Y$ et al. A review of magnetic resonance imaging and diffusion tensor imaging findings in mild traumatic brain injury. Brain Imaging Behav. 2012;6(2):137-192. https://doi.org/10.1007/s11682-012-9156-5

18. Huisman TA, Schwamm LH, Schaefer PW, Koroshetz WJ, Shetty-Alva N, Ozsunar Y et al. Diffusion tensor imaging as potential biomarker of white matter injury in diffuse axonal injury. AJNR Am J Neuroradiol. 2004 Mar;25(3):370-6.

19. Salmond CH, Menon DK, Chatfield DA, Williams GB, Pena A, Sahakian BJ et al. Diffusion tensor imaging in chronic head injury survivors: correlations with learning and memory indices. Neuroimage. 2006 Jan;29(1):117-24. https://doi.org/10.1016/j.neuroimage.2005.07.012

20. Le Bihan D, lima M. Diffusion magnetic resonance imaging: what water tells us about biological tissues. PLoS Biol. 2015 Jul;13(7):e1002203.https://doi.org/10.1371/journal.pbio.1002203

21. Beaulieu C. The basis of anisotropic water diffusion in the nervous system: a technical review. NMR Biomed. 2002 Nov-Dec;15(7-8):435-55. https://doi.org/10.1002/nbm.782
22. Karaarslan E, Arslan A. Diffusion weighted MR imaging in non-infarct lesions of the brain. Eur J Radiol. 2008 Mar;65(3):402-16. https://doi.org/10.1016/j.ejrad.2007.04.023 PMID:17555903

23. Le Bihan D. Apparent diffusion coefficient and beyond: what diffusion MR imaging can tell us about tissue structure. Radiology. 2013 Aug;268(2):318-22. https://doi.org/10.1148/radiol.13130420

24. Ogura A, Hatano I, Osakabe K, Yamaguchi N, Koyama D, Watanabe H. Importance of fractional b value for calculating apparent diffusion coefficient in DWI. AJR Am J Roentgenol. 2016 Dec;207(6):1239-43. https://doi.org/10.2214/AJR.15.15945

25. Mechtler LL, Shastri KK, Crutchfield KE. Advanced neuroimaging of mild traumatic brain injury. Neurol Clin. 2014 Feb;32(1):31-58. https://doi.org/10.1016/j.ncl.2013.08.002

26. Nucifora PG, Verma R, Lee SK, Melhem ER. Diffusion-tensor MR imaging and tractography: exploring brain microstructure and connectivity. Radiology. 2007 Nov;245(2):367-84. https://doi.org/10.1148/radiol.2452060445

27. Mori S, Zhang J. Principles of diffusion tensor imaging and its applications to basic neuroscience research. Neuron. 2006 Sep;51(5):527-39. https://doi.org/10.1016/j.neuron.2006.08.012

28. Pierpaoli C, Basser PJ. Toward a quantitative assessment of diffusion anisotropy. Magn Reson Med. 1996 Dec;36(6):893-906. https://doi.org/10.1002/mrm.1910360612

29. Basser PJ, Pajevic S, Pierpaoli C, Duda J, Aldroubi A. In vivo fiber tractography using DT-MRI data. Magn Reson Med. 2000 Oct;44(4):625-32.https://doi.org/10.1002/15222594(200010)44:4<625::AID-MRM17>3.0.CO;2-0

30. Winston GP.The physical and biological basis of quantitative parameters derived from diffusion MRI. Quant Imaging Med Surg. 2012 Dec;2(4):254-65. https://doi.org/10.3978/j.issn.2223-4292.2012.12.05

31. Song SK, Sun SW, Ramsbottom MJ, Chang C, Russell J, Cross AH. Dysmyelination revealed through MRI as increased radial (but unchanged axial) diffusion of water. Neuroimage. 2002 Nov;17(3):142936. https://doi.org/10.1006/nimg.2002.1267 PMID:12414282

32. Song SK, Sun SW, Ju WK, Lin SJ, Cross AH, Neufeld AH. Diffusion tensor imaging detects and differentiates axon and myelin degeneration in mouse optic nerve after retinal ischemia. Neuroimage. 2003 Nov;20(3):1714-22. https://doi.org/10.1016/j.neuroimage.2003.07.005

33. Garin-Muga A, Borro D. Review and challenges of brain analysis through DTI measurements. Stud Health Technol Inform. 2014;207:27-36. https://doi.org/10.3233/978-1-61499-474-9-27

34. Soares JM, Marques P, Alves V, Sousa N. A hitchhiker's guide to diffusion tensor imaging. Front Neurosci. 2013 Mar;7(31):31. https://doi.org/10.3389/fnins.2013.00031

35. Snook L, Plewes C, Beaulieu C. Voxel based versus region of interest analysis in diffusion tensor imaging of neurodevelopment. Neuroimage. 2007 Jan;34(1):243-52. https://doi.org/10.1016/j.neuroimage.2006.07.021

36. Mac Donald CL, Dikranian K, Bayly P, Holtzman D, Brody D. Diffusion tensor imaging reliably detects experimental traumatic axonal injury and indicates approximate time of injury. J Neurosci. 2007 Oct;27(44):11869-76. https://doi.org/10.1523/JNEUROSCI.3647-07.2007

37. Arfanakis K, Haughton VM, Carew JD, Rogers BP, Dempsey RJ, Meyerand ME. Diffusion tensor MR imaging in diffuse axonal injury. AJNR Am J Neuroradiol. 2002 May;23(5):794-802. https://doi.org/10.1016/j.neuroimage.2014.06.021

38. Edlow BL, Copen WA, Izzy S, Bakhadirov K, Kouwe A, Glenn MB et al. Diffusion tensor imaging in acute-to-subacute traumatic brain injury: a longitudinal analysis. BMC Neurol. 2016 Jan;16:2. https://doi.org/10.1186/s12883-015-0525-8

39. Mori S, van Zijl PC. Fiber tracking: principles and strategies - a technical review. NMR Biomed. 2002 Nov-Dec;15(7-8):468-80. https://doi.org/10.1002/nbm.781 
40. Tournier JD, Mori S, Leemans A. Diffusion tensor imaging and beyond. Magn Reson Med. 2011 Jun;65(6):1532-56. https://doi.org/10.1002/mrm.22924

41. Wang JY, Bakhadirov K, Devous MD Sr, Abdi H, McColl R, Moore $\mathrm{C}$ et al. Diffusion tensor tractography of traumatic diffuse axonal injury. Arch Neurol. 2008 May;65(5):619-26. https://doi.org/10.1001/archneur.65.5.619

42. Rutgers DR, Toulgoat F, Cazejust J, Fillard P, Lasjaunias P, Ducreux D. White matter abnormalities in mild traumatic brain injury: a diffusion tensor imaging study. AJNR Am J Neuroradiol. 2008 Mar;29(3):514-9. https://doi.org/10.3174/ajnr.A0856

43. Brandstack N, Kurki T, Tenovuo O. Quantitative diffusiontensor tractography of long association tracts in patients with traumatic brain injury without associated findings at routine MR imaging. Radiology. $2013 \mathrm{Apr}$;267(1):231-9. https://doi.org/10.1148/radiol.12112570

44. Yendiki A, Panneck P, Srinivasan P, Stevens A, Zöllei L, Augustinack $J$ et al. Automated probabilistic reconstruction of white-matter pathways in health and disease using an atlas of the underlying anatomy. Front Neuroinform. 2011 Oct;5:23. https://doi.org/10.3389/fninf.2011.00023

45. Koch SB, van Zuiden M, Nawijn L, Frijling JL, Veltman DJ, Olff M. Decreased uncinate fasciculus tract integrity in male and female patients with PTSD: a diffusion tensor imaging study. J Psychiatry Neurosci. 2017 Sep;42(5):331-42. https://doi.org/10.1503/jpn.160129

46. Smith SM, Jenkinson M, Johansen-Berg H, Rueckert D, Nichols TE, Mackay CE et al. Tract-based spatial statistics: voxelwise analysis of multi-subject diffusion data. Neuroimage. $2006 \mathrm{Jul}$;31(4):1487-505. https://doi.org/10.1016/j.neuroimage.2006.02.024

47. Bach M, Laun FB, Leemans A, Tax CM, Biessels GJ, Stieltjes $B$ et al. Methodological considerations on tract-based spatial statistics (TBSS). Neuroimage. 2014 Oct;100:358-69. https://doi.org/10.1016/j.neuroimage.2014.06.021

48. Zhang H, Yushkevich PA, Alexander DC, Gee JC. Deformable registration of diffusion tensor $\mathrm{MR}$ images with explicit orientation optimization. Med Image Anal. 2006 Oct;10(5):764-85. https://doi.org/10.1016/j.media.2006.06.004

49. Andrade CS, Conceição DM, Grassi DC et al. Evaluation of white matter integrity with tract-based spatial statistics in patients with traumatic brain injury. Vienna: European Society of Radiology; 2017. https://doi.org/10.1594/ecr2017/C-0747

50. Chu Z, Wilde EA, Hunter JV, McCauley SR, Bigler ED, Troyanskaya $M$ et al. Voxel-based analysis of diffusion tensor imaging in mild traumatic brain injury in adolescents. AJNR Am J Neuroradiol. 2010 Feb;31(2):340-6. https://doi.org/10.3174/ajnr.A1806

51. Lipton ML, Gellella E, Lo C, Gold T, Ardekani BA, Shifteh Ket al. Multifocal white matter ultrastructural abnormalities in mild traumatic brain injury with cognitive disability: a voxelwise analysis of diffusion tensor imaging. J Neurotrauma. 2008 Nov;25(11):1335-42. https://doi.org/10.1089/neu.2008.0547

52. Xu J, Rasmussen IA, Lagopoulos J, Håberg A. Diffuse axonal injury in severe traumatic brain injury visualized using high-resolution diffusion tensor imaging. J Neurotrauma. 2007 May;24(5):753-65. https://doi.org/10.1089/neu.2006.0208 